King's might well allow direct visualization of a suspect heart valve and thereby confirm the presence of vegetations, since its effective length of about a metre should suffice to reach both aortic and mitral valves. The present objection to invasive techniques in the suspected case of bacterial endocarditis would no longer apply, as this instrument would allow direct visualization (by retrograde routing via the brachial artery) of an affected valve before damaging intracardiac advancement could occur. The amount of visual interference caused by fluid blood in the visual field could probably be considerably cleared by the Valsalva manoeuvre when the endocardioscope is in the vicinity of a valve.

Unless such an instrument has already been tried and proved unsatisfactory, or unless an improved version of the above proposed instrument is already under trial, its necessity can be underlined by the experience of Dr. Reginald Hudson (personal communication, 1972), who has estimated from personal necropsies that about onethird of fatal cases were undiagnosed in life. He also considered that well over half such cases were missed in this country in general. Perhaps the plea of the research team at King's for further funds to carry on their excellent work may be answered.-I am, etc.,

Department of General Medicine, East Glamorgan General

J. DE SWIET

\section{Discriminant Value of Thyroid Function Tests}

SIR,-In the article by Dr. David B. Barnett and others (21 April, p. 144) linear discriminant analysis was used to calculate two straight lines which, in a Cartesian plot of T-3 uptake (Triosorb) and protein-bound iodine, served as the best boundary lines separating a euthyroid population from hypo- and hyperthyroid ones.

During the past two years in this laboratory we have used the combination of total thyroxine concentration in serum $(\mathrm{T}-4)$ and T-3 uptake (Triosorb) as the screening procedure for dysthyroidism. Besides giving the actural results for each test, the report forms display them as a point in a system of $\infty^{-}$ ordinates (see figure). The two curved

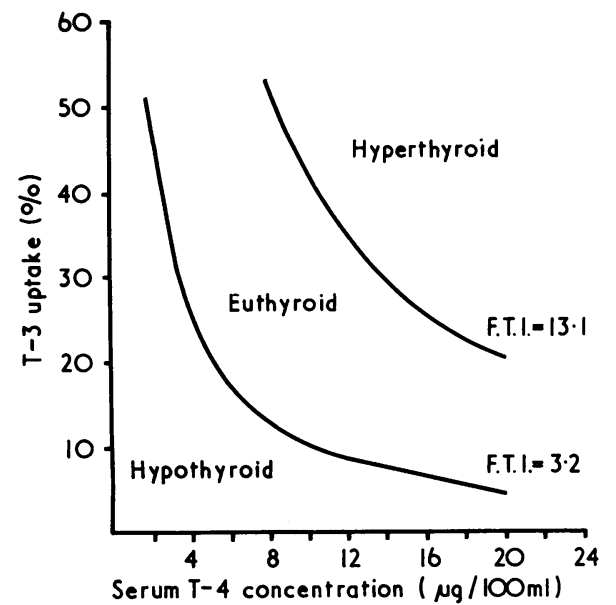

boundary lines in our graph have a location corresponding to the straight lines calculated by Dr. Barnett's group, but our lines repre- sent upper and lower limits for a free thyroxine index calculated as: F.T.I. $=(T-3$ uptake $\times \mathrm{T}-4) \div \mathrm{k}$

From table II in the authors' paper it can be seen that linear discriminant analysis of serum protein-bound iodine and T-3 uptake correctly identified 176 patients out of 191 By the free thyroxine index, however, only 168 of 191 patients were correctly allocated. From these data the authors postulate that their boundary lines have a greater discriminatory power than a free thyroxine index calculated on the basis of serum protein-bound iodine and T-3 uptake. However, these differences are not statistically significant $(P>0.05)$. Therefore it still remains to be shown whether linear discriminan analysis is superior to free thyroxine index in this respect-but certainly, this problem is subtle in comparison to the problem of supplying the clinician with self-explanatory laboratory data-for example, in the form of "cartoons."-I am, etc.,

J. VESTERDAL JøRGENSEN

Nuclear Medicine Laboratory,

Frederiksborg County Hospital

\section{Irradiation of C.N.S. in Leukaemia}

SIR,-In dealing with cancer radiation oncologists, as well as their medical confreres, are often pressured into giving treatment on the grounds that subclinical disease is present without adequate evidence of benefi or consideration of the hazards which may occur. In the case of prophylactic irradiation of the central nervous system, this is nowhere more obvious. Your recent leading article (19 May, p. 377) regarding the place of irradiation of the C.N.S. in acure lymphatic leukaemia, as well as the report of the Medical Research Council,' would appear to give the impression that the case for prophylactic C.N.S. irradiation has been proved. I would therefore like to enter through your journal some words of caution as well as criticism of the method of presentation.

Four questions need to be answered in the debate over prophylactic versus therapeutic radiation of the C.N.S. in acute ymphatic leukaemia.

(1) Does prophylactic treatment of the C.N.S. influence survival? At a recent meeting in Regina, Saskatchewan, Dr. Luis Borella, of the Pinkel Group in Memphis, updated the figures for survival in the two groups of their study VI. ${ }^{23}$ This was a controlled trial. At present 30 patients out of $45(66 \%)$ are still alive in the prophylactically treated group and 29 of the 49 $(60 \%)$ are alive in the non-prophylactic group. The corresponding figures in the M.R.C. study are not quoted.

(2) Does prophylactic C.N.S. therapy influence the haematological relapse rate? The figures currently quoted by Dr. Borella are that the number of patients still in the initial haematological remission is 28 out of 45 $(62 \%)$ in the prophylactic group and 27 out of $49(55 \%)$ in the group receiving no prophylaxis. The current M.R.C. figures indicate that to date the haematological relapse rate is greater in the prophylactic group.

(3) Does prophylactic C.N.S. therapy influence the control of C.N.S. leukaemia?
Pinkel and his associates ${ }^{2} 3$ appeared to show that treatment when C.N.S. leukaemia develops is not as effective as prophylaxis. In their study there were three patients out of $45(6.7 \%)$ treated prophylactically who developed subsequent evidence of C.N.S. disease at any time after the initial therapy. Five patients out of $25(20 \%)$ treated when C.N.S. disease developed had further evidence of C.N.S. disease. It should be pointed out, however, that there were 47 patients at risk (two were receiving radiation at the time of their report) and for adequate comparison the whole group should be taken into consideration when evaluating this form of therapy. Because of this, the effective recurrence rate is five out of 47 , or $10.6 \%$. This is again not significantly different from the prophylactic group. Unfortunately, I have been unable to get any up-to-date figures on the number of patients with C.N.S. relapse in either group. It is, however, known at this time that in the control group 33 patients have developed C.N.S. leukaemia-that is, one-third of the patients have not required therapy for C.N.S. disease. The corresponding figures in the M.R.C. study are again not stated.

(4) Does prophylactic C.N.S. therapy harm the patient? This is, as yet, unanswered. However, in the M.R.C. study four patients in the prophylactic group have died while in complete remission. The Memphis group have shown no difference in that three out of 45 in the prophylactic group and two out of 49 in the non-prophylactic group died in their initial complete remission. The paper of Campbell et al." shows an increased immunosuppression in those patients receiving prophylactic therapy. The potential late effects of radiation cannot be discounted, particularly when we are now thinking in terms of cure of patients with acute leukaemia. There may or may not be impairment of bone growth, though the factor of impairment of soft tissue growth has not, to my knowledge, been studied. The possibility of radiation carcinogenesis is definitely still present and the risk of this complication will be fairly high if the association of thymic radiation and carcinoma of the thyroid is any guide. Follow-up to date is, of course, too short to analyse the potential radiation carcinogenesis.

Since some $20 \%$ to $50 \%$ of patients with acute lymphoblastic leukaemia will not develop C.N.S. disease, are the risks in terms of morbidity and impairment of adequate chemotherapy sufficient to justify routine prophylactic radiation therapy? I would submit that this has not been proved at present. The current M.R.C. study should hopefully provide some of the answers.

I would suggest on the basis of the above figures that the C.N.S. relapse rate and haematological relapse rate be kept separate and distinct. The integration of the various relapse rates only confuses the issue. More emphasis should be placed on identifying the patients at greatest risk of developing C.N.S. involvement.-I am, etc.,

D. G. McGowaN

Dr. W. W. Cross Cancer Institute,

Edmonton, Alberta

1 Medical Research Council, British Medical fournal,

2 Aur, R. J. A., Simone, J. V., Hustu, H. C., and Verzosa, M.' S., Cancer, 1972, 29, 381.

3 Aur, R. J. A., et al., Blood, 1971, 37.272 1973, 2, 385 . 